The Agriculturists 13(2): 120-132(2015) ISSN 2304-7321 (Online), ISSN 1729-5211 (Print)

A Scientific Journal of Krishi Foundation

Indexed Journal

\title{
Yield Potential of Twelve Potato (Solanum tuberosum) Varieties Grown from Different Generations of Seed
}

\author{
Shafayet Hossain ${ }^{1}$, Golam Rasul ${ }^{2}$, M. A. Khaleque Mian², M. Moynul Haque ${ }^{3}$ \\ and Abdul Mannan Akanda ${ }^{4}$ \\ ${ }^{1}$ Bangladesh Agricultural Development Corporation, Krishi Bhaban, 49-51 Dilusha C/A, Dhaka 1000; \\ ${ }^{2}$ Depts. of Genetics and Plant Breeding, ${ }^{3}$ Agronomy and ${ }^{4}$ Plant Pathology, Bangabandhu Sheikh \\ Mujibur Rahman Agricultural University, Gazipur, Bangladesh \\ *Corresponding author and Email: shafayetbadc@ gmail.com
}

Received: 10 July 2014

Accepted: 12 December 2015

\begin{abstract}
An experiment was conducted at the research field of Bangabandhu Sheikh Mujibur Rahman Agricultural University (BSMRAU), Gazipur to determine the yield potential of twelve potato varieties for consecutive years during 2009-2011. The mean performance of varieties, generations and their interactions varied significantly. Field performance revealed that the plant height decreased in all the varieties Raja, Arinda, Patrones, Multa, Asterix, Granola, Felsina, Diamant, Cardinal, Provento, Ailsa and BARI TPS 1 in the $4^{\text {th }}$ generation compared to $2^{\text {nd }}$ generation (Breeders' Seed) except Raja and Arinda and it decreased compared to $3^{\text {nd }}$ generation in Patrones and Felsina. The highest plant vigor was observed in Felsina and Asterix in the $1^{\text {st }}$ generation, Patrones, Multa and Asterix in the $2^{\text {nd }}$ generation and Raja and Granola in the $3^{\text {rd }}$ generation. The highest number of stems per hill was produced by Patrones in all the three generations and the lowest was in Arinda in the $3^{\text {rd }}$ generation. The highest number of hills per plot was recorded in Asterix and Granola and the lowest were in Ailsa and Raja. Higher tuber yield per hill was obtained from Ailsa, followed by Raja, Arinda and Felsina. Patrones produced higher number of tubers per plot in all the three generations while Ailsa produced the minimum which were followed by Felsina, Diamant, Raja and Arinda in all the generations. The yield of different varieties in the $2^{\text {nd }}$ generation ranged from $11.94 \mathrm{t} / \mathrm{ha}$ (Ailsa) to $26.18 \mathrm{t} / \mathrm{ha}$ (Asterix) with an average yield of $19.06 \mathrm{t} / \mathrm{ha}$. However, the highest yield was observed by Asterix followed by Granola, Arinda, Provento and Diamant in the $2^{\text {nd }}$ generation.
\end{abstract}

Keywords: Potato varieties, yield potential, generation

\section{Introduction}

Among all the crops in the world, potato (Solanum tuberosum) is the most important one in respect of yield potential. Potentiality of any crop is determined by its genetic makeup. The production of protein and calorie per unit area and time is higher as compared to other major food crops including rice and wheat
(Pushkarnath, 1976). Now, it occupies the second place in acreage and production after rice in Bangladesh (Anonymous, 2010). Better varieties with integrated crop management practices will increase yield and provide higher productivity for the farmers. There are significant opportunities for improved potato production to address poverty, food security and 
environmental degradation. The total production of potato in the world was 315.10 million tons with an average yield was $16.73 \mathrm{t} / \mathrm{ha}$ in the year 2006. In Bangladesh, the production was 7.93 million tons in 2010 (Anonymous, 2010).

The yield per hectare was 35.26 tons in Denmark, 40.19 ton in France, 36.57 tons in Germany, 41.67 tons in the Netherlands and 43.67 tons in USA in 2006 (Anonymous, 2008). This indicates the high yield potential of potato crop. The yield of potato per ha in Bangladesh is lower compared to those in many potato growing countries of the world (BBS, 2010). The reasons for such a low yield and non-profitability of potato cultivation include lack of quality seeds of desired varieties, unavailability of certified seeds, lack of improved production methods, use of low yield potential cultivars, high production cost and short growing season. Out of the 42 varieties released by TCRC of Bangladesh Agricultural Research Institute, only a few are being cultivated by the farmers due to some reasons. The most important reasons for non acceptability of these varieties are susceptibility to the diseases and pests (Hossain, 2010), low yield and non-availability of quality seed tubers (Choudhury et al., 1995).

Yield can be increased considerably using high yield potential cultivars. Generally, the yield of exotic varieties is higher in their first generation (TCRC, 1988). The total seed potato requirement of Bangladesh is estimated at about 7.5 lakh tons per annum. Most of the seeds used are not of high quality. The farmers generally use the tubers for their own consumption as seeds, which results in poor yield in the following season. The locally produced certified seed or Truthfully Labelled Seed (TLS) meets only about $6.0 \%$ of the total requirement. For that reason, every year about 10,000 tons of potato seeds need to be imported spending huge amount of foreign currency (Hossain, 2010). As per seed potato production system, Breeder seed ( $2^{\text {nd }}$ generation) are produced by BARI which is used for production of Foundation seed ( $3^{\text {rd }}$ generation) by BADC.
The Foundation seed is ultimately used for production of Certified seed ( $4^{\text {th }}$ generation) and or Truthfully Labelled Seed (TLS) to be used at farmers' level. The Breeder seeds possess the highest genetic purity and yield potentiality, which declines with advancing generations. The increase or decrease in yield over generations gives an idea about the yield potentiality of a variety but the information on the yield potential of different potato varieties is insufficient. In this context, an investigation was undertaken to study the field performance and yields of twelve potato varieties recommended for cultivation in Bangladesh over the generations and to identify the low degenerated potato varieties.

\section{Materials and Methods}

The study was conducted at the experimental field of Bangabandhu Sheikh Mujibur Rahman Agricultural University (BSMRAU), Gazipur. Breeders seeds of twelve potato varieties namely Diamant, Cardinal, Granola, Patrones, Provento, Multa, Raja, Asterix, Felsina, Arinda, Ailsa and BARI TPS 1 were obtained from Breeders Seed Production Centre, BARI, Debigonj, Panchagarh for the study.

The study was conducted consecutively for three years. Breeder Seed was planted in 2008-09, third generation seed tubers foundation seed obtained from breeder seed were planted in 2009-10 and the 4th generation seed potato tubers or certified seed obtained from third generation were planted in 2010-11. The plant spacing was $60 \mathrm{~cm}$ from row to row and $15 \mathrm{~cm}$ from tuber to tuber. The unit plots were 4 $\mathrm{m} \times 2.4 \mathrm{~m}\left(9.6 \mathrm{~m}^{2}\right)$. Each plot contained 104 hills at the rate of 26 per row. The seed tubers were planted in November in the first, second and third years, respectively.

The experiment was laid out in a Randomized Complete Block Design with three replications in each generation. Data were recorded on growth and yield. Collected data were transformed into square roots and analyzed with MSTAT-C programme following two factors 
RCBD. The mean separation was done by Duncan's New Multiple Range Test at 5\% level of significance.

\section{Results and Discussion}

\subsection{Morphological characters}

The morphological characters of plants distinctly varied among the generations and varieties. The highest plant height was recorded in $2^{\text {nd }}$ generation which was similar to $4^{\text {th }}$ generation and decreased in $3^{\text {rd }}$ generation (Table 1). Plant height varied significantly among the varieties. Provento attained the tallest height of $35.30 \mathrm{~cm}$,
$42.92 \mathrm{~cm}, 52.38 \mathrm{~cm}$ and $57.90 \mathrm{~cm}$ at $35,40,45$ and 50 days after planting (DAP), respectively. This was followed by Asterix $(32.43 \mathrm{~cm}$ at 35 DAP, $48.48 \mathrm{~cm}$ at 45 Days after planting (DAP) and $52.97 \mathrm{~cm}$ at $50 \mathrm{DAP})$ and Felsina $(37.78 \mathrm{~cm}$ at 40 DAP). At 50 DAP, the shortest plant height $(35.49 \mathrm{~cm})$ was observed in Arinda was similar to Patrones (Table 2), Ailsa $(36.52 \mathrm{~cm}$ ) and Granola $(38.30 \mathrm{~cm})$. Interaction of variety and generations revealed that the tallest plants were observed in $2^{\text {nd }}$ generation of Felsina $(64.37 \mathrm{~cm})$ followed by $2^{\text {nd }}$ generation of Provento (62.67 $\mathrm{cm})$ and $4^{\text {th }}$ generation of Provento $(62.07 \mathrm{~cm})$ at 50 DAP.

Table 1. Average plant height recorded in three successive generations

\begin{tabular}{ccccc}
\hline \multirow{2}{*}{ Generation } & \multicolumn{4}{c}{ plant height $(\mathrm{cm})$} \\
\cline { 2 - 5 } & $35 \mathrm{DAP}$ & $40 \mathrm{DAP}$ & $45 \mathrm{DAP}$ & 50DAP \\
\hline $2^{\text {nd }}$ gen. $\left(\mathrm{G}_{2}\right)$ & $31.48 \mathrm{a}$ & $35.09 \mathrm{a}$ & $43.90 \mathrm{a}$ & $47.77 \mathrm{a}$ \\
$3^{\text {rd }}$ gen. $\left(\mathrm{G}_{3}\right)$ & $19.33 \mathrm{c}$ & $26.15 \mathrm{~b}$ & $36.24 \mathrm{c}$ & $39.54 \mathrm{~b}$ \\
$4^{\text {th }}$ gen. $\left(\mathrm{G}_{4}\right)$ & $29.81 \mathrm{~b}$ & $35.92 \mathrm{a}$ & $40.82 \mathrm{~b}$ & $46.38 \mathrm{a}$ \\
\hline $\mathrm{CV}(\%)$ & 10.00 & 12.21 & 10.22 & 8.52 \\
\hline
\end{tabular}

Figures with the same letter(s) in column are not significantly different.

Table 2. Performance of different varieties for plant height

\begin{tabular}{lllll}
\hline \multirow{2}{*}{ Varieties } & \multicolumn{4}{c}{ Plant height $(\mathrm{cm})$} \\
\cline { 2 - 5 } & $35 \mathrm{DAP}$ & $40 \mathrm{DAP}$ & $45 \mathrm{DAP}$ & $50 \mathrm{DAP}$ \\
\hline Diamant & $27.40 \mathrm{de}$ & $32.43 \mathrm{de}$ & $39.71 \mathrm{de}$ & $43.50 \mathrm{de}$ \\
Patrones & $25.27 \mathrm{e}$ & $28.63 \mathrm{ef}$ & $33.19 \mathrm{gh}$ & $36.02 \mathrm{f}$ \\
Raja & $22.46 \mathrm{f}$ & $29.61 \mathrm{ef}$ & $37.78 \mathrm{ef}$ & $42.11 \mathrm{e}$ \\
BARI TPS 1 & $25.62 \mathrm{e}$ & $31.43 \mathrm{de}$ & $43.55 \mathrm{~cd}$ & $47.70 \mathrm{c}$ \\
Arinda & $17.18 \mathrm{~g}$ & $23.12 \mathrm{~g}$ & $30.57 \mathrm{~h}$ & $35.49 \mathrm{f}$ \\
Provento & $35.30 \mathrm{a}$ & $42.92 \mathrm{a}$ & $52.38 \mathrm{a}$ & $57.90 \mathrm{a}$ \\
Felsina & $30.13 \mathrm{bc}$ & $37.78 \mathrm{bc}$ & $47.34 \mathrm{bc}$ & $52.00 \mathrm{~b}$ \\
Multa & $31.59 \mathrm{~b}$ & $32.47 \mathrm{de}$ & $41.98 \mathrm{de}$ & $45.91 \mathrm{~cd}$ \\
Asterix & $32.43 \mathrm{~b}$ & $39.37 \mathrm{ab}$ & $48.48 \mathrm{~b}$ & $52.97 \mathrm{~b}$ \\
Granola & $25.93 \mathrm{de}$ & $29.16 \mathrm{ef}$ & $34.94 \mathrm{fg}$ & $38.30 \mathrm{f}$ \\
Cardinal & $28.57 \mathrm{~cd}$ & $35.03 \mathrm{~cd}$ & $41.05 \mathrm{de}$ & $46.33 \mathrm{~cd}$ \\
Ailsa & $20.64 \mathrm{f}$ & $26.67 \mathrm{fg}$ & $32.88 \mathrm{gh}$ & $36.52 \mathrm{f}$ \\
\hline CV $(\%)$ & 10.0 & 12.21 & 10.22 & 8.52 \\
\hline
\end{tabular}

Figures with the same letter(s) in column are not significantly different. 
Table 3. Interaction effect of variety and generations on plant height

\begin{tabular}{|c|c|c|c|c|c|}
\hline \multirow{2}{*}{\multicolumn{2}{|c|}{ Generation $\mathrm{x}$ variety }} & \multicolumn{4}{|c|}{ Plant height $(\mathrm{cm})$} \\
\hline & & 35 DAP & 40 DAP & 45 DAP & 50 DAP \\
\hline \multirow{12}{*}{$\mathrm{G}_{2}$} & Diamant & $32.43 \mathrm{~b}-\mathrm{d}$ & $38.20 \mathrm{c}-\mathrm{f}$ & $44.73 \mathrm{c}-\mathrm{e}$ & $47.27 \mathrm{~d}-\mathrm{g}$ \\
\hline & Patrones & $28.63 \mathrm{~d}-\mathrm{g}$ & $31.10 \mathrm{f}-1$ & $35.24 \mathrm{~g}-1$ & $36.93 \mathrm{i}-\mathrm{n}$ \\
\hline & Raja & $22.97 \mathrm{~h}-\mathrm{k}$ & $28.15 \mathrm{gm}$ & $38.35 \mathrm{~d}-\mathrm{k}$ & $42.07 \mathrm{e}-\mathrm{k}$ \\
\hline & BARI TPS 1 & $29.97 c-f$ & $33.37 \mathrm{e}-\mathrm{j}$ & $46.81 \mathrm{bc}$ & $52.77 \mathrm{~cd}$ \\
\hline & Arinda & $19.67 \mathrm{k}-\mathrm{m}$ & $25.20 \mathrm{k}-\mathrm{m}$ & $35.57 \mathrm{f}-1$ & $41.13 \mathrm{~g}-\mathrm{m}$ \\
\hline & Provento & $41.63 \mathrm{a}$ & $45.90 \mathrm{ab}$ & $57.95 \mathrm{a}$ & $62.67 \mathrm{ab}$ \\
\hline & Felsina & $40.23 \mathrm{a}$ & $45.93 \mathrm{ab}$ & $57.81 \mathrm{a}$ & $64.37 \mathrm{a}$ \\
\hline & Multa & $34.87 \mathrm{bc}$ & $35.33 \mathrm{~d}-\mathrm{g}$ & 44.00 cde & $47.17 \mathrm{~d}-\mathrm{g}$ \\
\hline & Asterix & $41.40 \mathrm{a}$ & $44.80 \mathrm{abc}$ & $53.71 \mathrm{ab}$ & $56.40 \mathrm{bc}$ \\
\hline & Granola & $31.30 \mathrm{~b}-\mathrm{e}$ & $32.27 \mathrm{e}-\mathrm{k}$ & $40.82 \mathrm{c}-\mathrm{i}$ & $44.47 \mathrm{e}-\mathrm{h}$ \\
\hline & Cardinal & $32.40 \mathrm{~b}-\mathrm{d}$ & $34.67 \mathrm{~d}-\mathrm{i}$ & $40.51 \mathrm{c}-\mathrm{i}$ & $43.73 \mathrm{e}-\mathrm{i}$ \\
\hline & Ailsa & $22.27 \mathrm{i}-1$ & $26.11 \mathrm{j}-\mathrm{m}$ & $31.37 \mathrm{jkl}$ & $34.27 \mathrm{lmn}$ \\
\hline \multirow{12}{*}{$\mathrm{G}_{3}$} & Diamant & $20.50 \mathrm{j}-\mathrm{m}$ & $23.60 \mathrm{lmn}$ & $33.11 \mathrm{i}-1$ & $36.24 j-n$ \\
\hline & Patrones & $21.67 \mathrm{jkl}$ & $26.93 \mathrm{i}-\mathrm{m}$ & $32.23 \mathrm{jkl}$ & $35.43 \mathrm{k}-\mathrm{n}$ \\
\hline & Raja & $16.47 \mathrm{mn}$ & $22.37 \mathrm{mn}$ & $31.95 \mathrm{jkl}$ & $36.08 \mathrm{j}-\mathrm{n}$ \\
\hline & BARI TPS1 & $17.80 \mathrm{lmn}$ & $26.03 \mathrm{j}-\mathrm{m}$ & $43.31 \mathrm{c}-\mathrm{g}$ & $41.83 \mathrm{f}-\mathrm{k}$ \\
\hline & Arinda & 10.20 o & $16.83 n$ & $23.01 \mathrm{~m}$ & $26.08 \mathrm{o}$ \\
\hline & Provento & $21.93 \mathrm{i}-1$ & $33.37 \mathrm{e}-\mathrm{j}$ & 44.26 cde & $48.97 \mathrm{~d}-\mathrm{f}$ \\
\hline & Felsina & $23.23 \mathrm{~h}-\mathrm{k}$ & $33.03 \mathrm{e}-\mathrm{j}$ & $43.66 c-f$ & $47.86 \mathrm{~d}-\mathrm{g}$ \\
\hline & Multa & $24.60 \mathrm{~g}-\mathrm{k}$ & $30.00 \mathrm{~g}-\mathrm{j}$ & $38.96 \mathrm{c}-\mathrm{j}$ & $43.64 \mathrm{e}-\mathrm{i}$ \\
\hline & Asterix & $24.23 \mathrm{~g}-\mathrm{k}$ & $35.33 \mathrm{~d}-\mathrm{g}$ & $47.12 \mathrm{bc}$ & $49.39 \mathrm{de}$ \\
\hline & Granola & $16.47 \mathrm{mn}$ & $22.00 \mathrm{mn}$ & $28.82 \mathrm{~lm}$ & 31.89 no \\
\hline & Cardinal & 20.97 jm & $29.23 \mathrm{gm}$ & $37.72 \mathrm{~d}-\mathrm{k}$ & $43.18 \mathrm{e}-\mathrm{j}$ \\
\hline & Ailsa & 13.93 no & $23.10 \mathrm{mn}$ & $30.70 \mathrm{kl}$ & $33.84 \mathrm{mn}$ \\
\hline \multirow{12}{*}{$\mathrm{G}_{4}$} & Diamant & $29.27 \mathrm{~d}-\mathrm{g}$ & $35.50 \mathrm{~d}-\mathrm{g}$ & $41.30 \mathrm{c}-\mathrm{h}$ & $47.00 \mathrm{~d}-\mathrm{g}$ \\
\hline & Patrones & $25.50 \mathrm{f}-\mathrm{j}$ & 27.87 g-m & $32.10 \mathrm{jkl}$ & $35.70 \mathrm{k}-\mathrm{n}$ \\
\hline & Raja & $27.93 \mathrm{~d}-\mathrm{h}$ & $38.30 \mathrm{c}-\mathrm{f}$ & $43.03 \mathrm{c}-\mathrm{h}$ & $48.17 \mathrm{~d}-\mathrm{g}$ \\
\hline & BARI TPS1 & $29.10 \mathrm{~d}-\mathrm{g}$ & 34.90 d-h & $40.53 \mathrm{c}-\mathrm{i}$ & $48.50 \mathrm{~d}-\mathrm{g}$ \\
\hline & Arinda & $21.67 \mathrm{jkl}$ & $27.33 \mathrm{hm}$ & $33.13 \mathrm{i}-1$ & 39.27 h-m \\
\hline & Provento & $42.33 \mathrm{a}$ & $49.50 \mathrm{a}$ & $54.93 \mathrm{a}$ & $62.07 \mathrm{ab}$ \\
\hline & Felsina & $26.93 \mathrm{e}-\mathrm{i}$ & $34.37 \mathrm{~d}-\mathrm{i}$ & $40.53 \mathrm{c}-\mathrm{i}$ & $43.77 \mathrm{e}-\mathrm{i}$ \\
\hline & Multa & $35.30 \mathrm{~b}$ & 40.07 b-e & $42.97 \mathrm{c}-\mathrm{h}$ & $46.93 \mathrm{~d}-\mathrm{g}$ \\
\hline & Asterix & $31.67 \mathrm{~b}-\mathrm{e}$ & $37.97 \mathrm{c}-\mathrm{f}$ & $44.60 \mathrm{cde}$ & $53.13 \mathrm{~cd}$ \\
\hline & Granola & $30.03 \mathrm{c}-\mathrm{f}$ & $33.20 \mathrm{e}-\mathrm{j}$ & 35.17 h-1 & 38.53 h-n \\
\hline & Cardinal & $32.33 \mathrm{~b}-\mathrm{d}$ & $41.20 \mathrm{~b}-\mathrm{d}$ & $44.93 \mathrm{~cd}$ & $52.07 \mathrm{~cd}$ \\
\hline & Ailsa & $25.70 \mathrm{f}-\mathrm{j}$ & $30.80 \mathrm{f}-1$ & $36.57 \mathrm{e}-1$ & $41.47 \mathrm{f}-1$ \\
\hline \multicolumn{2}{|c|}{$\mathrm{CV}(\%)$} & 10.00 & 12.21 & 10.22 & 8.52 \\
\hline
\end{tabular}

Figures with the same letter(s) in a column are not significantly different. 
The highest foliage coverage was in $4^{\text {th }}$ generation $(84.72 \%)$ which was similar to that in $3^{\text {rd }}$ generation (Table 3). Among varieties the highest foliage coverage was recorded in Asterix $(89.44 \%)$ and the lowest was in Ailsa (52.22\%). Interaction of variety and generations also revealed that the highest foliage coverage (\%) was in $2^{\text {nd }}$ generation of Asterix $(98.33 \%)$ followed by $2^{\text {nd }}$ generation of BARI TPS1 $(93.33 \%)$, and $2^{\text {nd }}$ generation of Felsina $(93.34 \%)$ and $4^{\text {th }}$ generation of Provento $(93.33 \%)$ at 50 DAP (Table 4). The highest plant vigor (5.00) was obtained in $2^{\text {nd }}$ generation of Felsina and Asterix, $4^{\text {th }}$ geneartion of Raja and Granola and the lowest was found in $2^{\text {nd }}$ generation of Ailsa (1.66) followed by $3^{\text {rd }}$ generation of Ailsa (2.33) and $4^{\text {th }}$ generation of Ailsa (2.66).
Among the generations, the highest number of stems per hill was produced in $2^{\text {nd }}$ generation followed by $3^{\text {rd }}$ and $4^{\text {th }}$ generations. A significant variation in number of stems per hill was observed among the varieties (Table 5). The highest number of stems per hill was produced by Patrones (5.20) followed by Multa (4.10), Diamant (4.06), Asterix (4.03) and Cardinal (3.89). The lowest number of stems (2.71) was produced by Arinda and Ailsa. Interaction of variety and generations revealed that the highest number of stems per hill was produced in $2^{\text {nd }}$ generation of Diamant (5.83) and was followed by Cardinal (5.63) and Patrones (5.60) in $2^{\text {nd }}$ generation and Patrones in $3^{\text {rd }}$ generation. The lowest number of stems (2.23) was obtained in Ailsa, Cardinal, Felsina and Provento in the $4^{\text {th }}$ generation (Table 6).

Table 4. Effect of generations on foliage coverage, plant vigor and stems/hill

\begin{tabular}{lccc}
\hline Generation & $\begin{array}{c}\text { Foliage coverage } \\
(\%)\end{array}$ & $\begin{array}{c}\text { Plant vigor } \\
(0-5)\end{array}$ & $\begin{array}{c}\text { Stems/hill } \\
\text { (no.) }\end{array}$ \\
\hline $\mathrm{G}_{2}$ & $82.08 \mathrm{a}$ & $4.17 \mathrm{ab}$ & $4.29 \mathrm{a}$ \\
$\mathrm{G}_{3}$ & $74.31 \mathrm{~b}$ & $4.01 \mathrm{~b}$ & $3.69 \mathrm{~b}$ \\
$\mathrm{G}_{4}$ & $84.72 \mathrm{a}$ & $4.35 \mathrm{a}$ & $2.81 \mathrm{c}$ \\
\hline $\mathrm{CV}(\%)$ & 10.44 & 9.6 & 10.04 \\
\hline
\end{tabular}

Figures with the same letter(s) in a column are not significantly different

Table 5. Performance of varieties in respect of foliage coverage, plant vigor and stems/hill

\begin{tabular}{lccc}
\hline Varieties & $\begin{array}{c}\text { Foliage coverage } \\
(\%)\end{array}$ & $\begin{array}{c}\text { Plant vigor } \\
(0-5)\end{array}$ & $\begin{array}{c}\text { Stems/hill } \\
(\text { no. })\end{array}$ \\
\hline Diamant & $83.33 \mathrm{a}$ & $4.39 \mathrm{ab}$ & $4.06 \mathrm{~b}$ \\
Patrones & $85.00 \mathrm{a}$ & $4.39 \mathrm{ab}$ & $5.20 \mathrm{a}$ \\
Raja & $73.89 \mathrm{c}$ & $4.17 \mathrm{~b}$ & $3.48 \mathrm{c}$ \\
BARI TPS1 & $85.56 \mathrm{a}$ & $4.50 \mathrm{ab}$ & $3.21 \mathrm{c}$ \\
Arinda & $73.89 \mathrm{bc}$ & $3.61 \mathrm{c}$ & $2.71 \mathrm{~d}$ \\
Provento & $84.44 \mathrm{a}$ & $4.56 \mathrm{ab}$ & $3.38 \mathrm{c}$ \\
Felsina & $82.22 \mathrm{ab}$ & $4.28 \mathrm{ab}$ & $3.14 \mathrm{c}$ \\
Multa & $87.22 \mathrm{a}$ & $4.28 \mathrm{ab}$ & $4.10 \mathrm{~b}$ \\
Asterix & $89.44 \mathrm{a}$ & $4.67 \mathrm{a}$ & $4.03 \mathrm{~b}$ \\
Granola & $85.56 \mathrm{a}$ & $4.67 \mathrm{a}$ & $3.30 \mathrm{c}$ \\
Cardinal & $81.67 \mathrm{abc}$ & $4.39 \mathrm{ab}$ & $3.89 \mathrm{~b}$ \\
Ailsa & $52.22 \mathrm{~d}$ & $2.22 \mathrm{~d}$ & $2.71 \mathrm{~d}$ \\
\hline CV(\%) & 10.44 & 9.60 & 10.04 \\
\hline
\end{tabular}

Figures with the same letter(s) in a column are not significantly different. 
Table 6. Interaction effect of variety and generations on foliage coverage, plant vigor and stems/hill

\begin{tabular}{|c|c|c|c|c|}
\hline \multicolumn{2}{|c|}{ Variety X Generations } & \multirow{2}{*}{$\begin{array}{c}\begin{array}{c}\text { Foliage coverage } \\
(\%)\end{array} \\
90.0 \text { a-c }\end{array}$} & \multirow{2}{*}{$\begin{array}{c}\begin{array}{c}\text { Plant vigor } \\
(0-5)\end{array} \\
4.66 \text { a-c }\end{array}$} & \multirow{2}{*}{$\begin{array}{c}\begin{array}{c}\text { Stems/hill } \\
\text { (no.) }\end{array} \\
5.83 \mathrm{a}\end{array}$} \\
\hline \multirow{12}{*}{$\mathrm{G}_{2}$} & Diamant & & & \\
\hline & Patrones & 83.33 a-f & $4.00 \mathrm{c}-\mathrm{f}$ & $5.60 \mathrm{ab}$ \\
\hline & Raja & $63.33 \mathrm{~h}$ & $3.66 \mathrm{e}-\mathrm{g}$ & $4.30 \mathrm{c}-\mathrm{e}$ \\
\hline & BARI TPS1 & 93.33 a & $4.66 \mathrm{a}-\mathrm{c}$ & $3.43 \mathrm{f}-\mathrm{i}$ \\
\hline & Arinda & $70.00 \mathrm{f}-\mathrm{h}$ & $3.00 \mathrm{~g}-\mathrm{i}$ & $3.00 \mathrm{~h}-1$ \\
\hline & Provento & $90.00 \mathrm{a}-\mathrm{c}$ & $4.83 \mathrm{ab}$ & $3.63 \mathrm{f}-\mathrm{h}$ \\
\hline & Felsina & 93.33 a & $5.00 \mathrm{a}$ & $3.73 \mathrm{e}-\mathrm{g}$ \\
\hline & Multa & $88.33 \mathrm{a}-\mathrm{d}$ & $4.33 \mathrm{a}-\mathrm{e}$ & $4.46 \mathrm{c}$ \\
\hline & Asterix & $98.33 \mathrm{a}$ & $5.00 \mathrm{a}$ & $5.10 \mathrm{~b}$ \\
\hline & Granola & $90.00 \mathrm{a}-\mathrm{c}$ & $4.83 \mathrm{ab}$ & $3.76 \mathrm{~d}-\mathrm{g}$ \\
\hline & Cardinal & $85.00 \mathrm{a}-\mathrm{f}$ & $4.33 \mathrm{a}-\mathrm{e}$ & $5.63 \mathrm{ab}$ \\
\hline & Ailsa & $40.00 \mathrm{i}$ & $1.66 \mathrm{j}$ & $3.06 \mathrm{~g}-\mathrm{k}$ \\
\hline \multirow{12}{*}{$\mathrm{G}_{3}$} & Diamant & $71.67 \mathrm{~d}-\mathrm{h}$ & $4.00 \mathrm{c}-\mathrm{f}$ & $3.63 \mathrm{e}-\mathrm{h}$ \\
\hline & Patrones & $88.33 \mathrm{a}-\mathrm{d}$ & $4.66 \mathrm{a}-\mathrm{c}$ & $5.56 \mathrm{ab}$ \\
\hline & Raja & $70.00 \mathrm{f}-\mathrm{h}$ & $3.83 \mathrm{~d}-\mathrm{f}$ & $3.20 \mathrm{f}-\mathrm{k}$ \\
\hline & BARI TPS1 & $73.33 \mathrm{c}-\mathrm{h}$ & $4.00 \mathrm{c}-\mathrm{f}$ & $3.50 \mathrm{f}-\mathrm{i}$ \\
\hline & Arinda & $66.67 \mathrm{gh}$ & $3.33 \mathrm{f}-\mathrm{h}$ & $3.26 \mathrm{f}-\mathrm{k}$ \\
\hline & Provento & $70.00 \mathrm{f}-\mathrm{h}$ & $4.00 \mathrm{c}-\mathrm{f}$ & $3.86 \mathrm{c}-\mathrm{f}$ \\
\hline & Felsina & $83.33 \mathrm{a}-\mathrm{f}$ & $4.50 \mathrm{a}-\mathrm{d}$ & $3.36 \mathrm{f}-\mathrm{j}$ \\
\hline & Multa & $91.67 \mathrm{ab}$ & $4.66 \mathrm{a}-\mathrm{c}$ & $4.40 \mathrm{~cd}$ \\
\hline & Asterix & 86.67 a-e & $4.66 \mathrm{a}-\mathrm{c}$ & $3.50 \mathrm{f}-\mathrm{i}$ \\
\hline & Granola & $75.00 \mathrm{~b}-\mathrm{h}$ & $4.16 \mathrm{~b}-\mathrm{e}$ & $3.46 \mathrm{f}-\mathrm{i}$ \\
\hline & Cardinal & $71.67 \mathrm{e}-\mathrm{h}$ & $4.00 \mathrm{c}-\mathrm{f}$ & $3.73 \mathrm{e}-\mathrm{g}$ \\
\hline & Ailsa & $43.33 \mathrm{i}$ & $2.33 \mathrm{i}$ & $2.86 \mathrm{i}-\mathrm{n}$ \\
\hline \multirow{12}{*}{$\mathrm{G}_{4}$} & Diamant & $88.33 \mathrm{a}-\mathrm{d}$ & $4.50 \mathrm{a}-\mathrm{d}$ & $2.70 \mathrm{~J}-\mathrm{n}$ \\
\hline & Patrones & $83.33 \mathrm{a}-\mathrm{f}$ & $4.50 \mathrm{a}-\mathrm{d}$ & $4.43 \mathrm{c}$ \\
\hline & Raja & $88.33 \mathrm{a}-\mathrm{d}$ & $5.00 \mathrm{a}$ & 2.93 h-m \\
\hline & BARI TPS1 & $90.00 \mathrm{a}-\mathrm{c}$ & $4.83 \mathrm{ab}$ & 2.70 j-n \\
\hline & Arinda & $85.00 \mathrm{a}-\mathrm{f}$ & $4.50 \mathrm{a}-\mathrm{d}$ & $1.86 \mathrm{o}$ \\
\hline & Provento & $93.33 \mathrm{a}$ & $4.83 \mathrm{ab}$ & $2.63 \mathrm{k}-\mathrm{n}$ \\
\hline & Felsina & $70.00 \mathrm{f}-\mathrm{h}$ & $3.33 \mathrm{f}-\mathrm{h}$ & $2.331-0$ \\
\hline & Multa & $81.67 \mathrm{a}-\mathrm{g}$ & $3.83 \mathrm{~d}-\mathrm{f}$ & $3.43 \mathrm{f}-\mathrm{i}$ \\
\hline & Asterix & $83.33 \mathrm{a}-\mathrm{f}$ & $4.33 \mathrm{a}-\mathrm{e}$ & $3.50 \mathrm{f}-\mathrm{i}$ \\
\hline & Granola & $91.67 \mathrm{ab}$ & $5.00 \mathrm{a}$ & $2.66 \mathrm{k}-\mathrm{n}$ \\
\hline & Cardinal & $88.33 \mathrm{a}-\mathrm{d}$ & $4.83 \mathrm{ab}$ & $2.30 \mathrm{~m}-\mathrm{o}$ \\
\hline & Ailsa & $73.33 \mathrm{c}-\mathrm{h}$ & $2.66 \mathrm{~h}$ & 2.20 no \\
\hline $\mathrm{CV}$ & & 10.44 & 9.6 & 10.04 \\
\hline
\end{tabular}

Figures with the same letter(s) in a column are not significantly different. 


\subsection{Yield and yield contributing characters}

A significant variation in respect of the number of hills per plot was observed among the varieties. The highest number of hills per plot was produced by Granola (89.44) followed by Asterix, BARI TPS1 and Diamant (Table 7). The lowest number of hills per plot was observed in the variety Ailsa. Interaction of variety and generation showed that the highest number of hills per plot was produced by Asterix and Granola in $2^{\text {nd }}$ generation. The lowest number of hills per plot was produced by Ailsa in all three generations. The number of hills per plot of all the varieties decreased in the $3^{\text {rd }}$ generation compared to $2^{\text {nd }}$ generation except Multa. The same trend was observed in the $4^{\text {th }}$ generation compared to $3^{\text {rd }}$ generation except Raja, Arinda and Ailsa (Table 8).

Significant variation in tuber yield per plot $(\mathrm{kg})$ was observed among the varieties and interaction of generations and varieties (Table 7). The highest yield of tuber per plot was $24.22 \mathrm{~kg}$ in Granola followed by Asterix $(23.36 \mathrm{~kg})$, Provento $(22.64 \mathrm{~kg})$ and Arinda $(22.01 \mathrm{~kg})$ and the lowest was obtained from Ailsa $(11.14 \mathrm{~kg})$. Interaction of generations and varieties revealed that the highest yield was produced by Granola $(25.64 \mathrm{~kg})$ in 2 nd generation followed by
Asterix, Arinda, Diamant and Provento $2^{\text {nd }}$ generation and the lowest yield was produced by Ailsa $(10.53 \mathrm{~kg})$ in the $3^{\text {rd }}$ generation $(10.53$ $\mathrm{kg}$ )(Table 8). Among the varieties, the highest number of tubers per plot was obtained from Patrones (678.6) followed by Granola (580.1), Asterix (541.8) and BARI TPS1 (531.1) and the lowest was in Ailsa (Table 7). Data on interaction of generations and varieties showed that the highest number of tubers per plot was obtained in Patrones $(849.7)$ during $2^{\text {nd }}$ generation and the lowest yield was produced by Ailsa (246.0) in the $3^{\text {rd }}$ generation (Table 8).

The highest yield ( $\mathrm{t} / \mathrm{ha}$ ) was produced by $2^{\text {nd }}$ generation followed by $4^{\text {th }}$ and $3^{\text {rd }}$ generation (Figure 1). The highest yield was obtained from Granola followed by Asterix and Provento and the lowest was found in Ailsa. Although, Ailsa had the highest yield per hill but the yield per hectare was the lowest due to fewer numbers of hills per plot. Out of twelve potato varieties Ailsa, Multa, Felsina and Raja yielded less than $20.0 \mathrm{t} / \mathrm{ha}$. The highest yield was obtained from Asterix in the $2^{\text {nd }}$ generation (26.18) followed by Granola in the $3^{\text {rd }}(23.92)$ and $4^{\text {th }}(24.73)$ generations, while the lowest was obtained from Ailsa in $2^{\text {nd }}(11.94)$ generation followed by $3^{\text {rd }}$ (10.96) and $4^{\text {th }}(11.92)$ generations (Figure 2).

Table 7. Performance of varieties on yield and yield contributing characters

\begin{tabular}{lcccc}
\hline Varieties & $\begin{array}{c}\text { Hill/ } \\
\text { Plot(no) }\end{array}$ & $\begin{array}{c}\text { Tuber yield/ } \\
\text { hill }(\mathrm{g})\end{array}$ & $\begin{array}{c}\text { Tuber yield/ } \\
\text { plot }(\mathrm{kg})\end{array}$ & $\begin{array}{c}\text { No. of tuber/ } \\
\text { Plot }\end{array}$ \\
\hline Diamant & $86.11 \mathrm{a}$ & $244.2 \mathrm{gh}$ & $20.91 \mathrm{de}$ & $437.6 \mathrm{c}$ \\
Patrones & $64.33 \mathrm{~d}$ & $325.5 \mathrm{c}$ & $20.94 \mathrm{de}$ & $678.6 \mathrm{a}$ \\
Raja & $25.33 \mathrm{f}$ & $713.1 \mathrm{~b}$ & $17.66 \mathrm{f}$ & $415.9 \mathrm{~cd}$ \\
BARI TPS1 & $88.11 \mathrm{a}$ & $227.9 \mathrm{~h}$ & $19.58 \mathrm{e}$ & $531.1 \mathrm{~b}$ \\
Arinda & $68.89 \mathrm{c}$ & $329.2 \mathrm{c}$ & $22.01 \mathrm{~b}-\mathrm{d}$ & $430.2 \mathrm{~cd}$ \\
Provento & $81.44 \mathrm{~b}$ & $289.8 \mathrm{~d}-\mathrm{f}$ & $22.64 \mathrm{bc}$ & $430.3 \mathrm{~cd}$ \\
Felsina & $59.44 \mathrm{e}$ & $300.1 \mathrm{c}-\mathrm{e}$ & $17.68 \mathrm{f}$ & $380.3 \mathrm{~d}$ \\
Multa & $71.67 \mathrm{c}$ & $260.0 \mathrm{f}-\mathrm{h}$ & $17.51 \mathrm{f}$ & $410.7 \mathrm{~cd}$ \\
Asterix & $89.11 \mathrm{a}$ & $276.9 \mathrm{e}-\mathrm{g}$ & $23.36 \mathrm{ab}$ & $541.8 \mathrm{~b}$ \\
Granola & $89.44 \mathrm{a}$ & $277.0 \mathrm{e}-\mathrm{g}$ & $24.22 \mathrm{a}$ & $580.1 \mathrm{~b}$ \\
Cardinal & $70.22 \mathrm{c}$ & $316.9 \mathrm{~cd}$ & $21.48 \mathrm{~cd}$ & $452.2 \mathrm{c}$ \\
Ailsa & $13.11 \mathrm{~g}$ & $850.6 \mathrm{a}$ & $11.14 \mathrm{~g}$ & $295.8 \mathrm{e}$ \\
\hline CV(\%) & 5.98 & 8.96 & 10.82 & 10.82 \\
\hline
\end{tabular}

Figures with the same letter(s) in a column are not significantly different. 
Table 8. Interaction effect of variety and generation on yield and yield contributing characters

\begin{tabular}{|c|c|c|c|c|c|}
\hline \multicolumn{2}{|c|}{ Variety $\mathrm{x}$ generation } & $\begin{array}{l}\text { No. of hill } \\
\text { /plot }\end{array}$ & $\begin{array}{c}\text { Tuber yield } \\
\text { /hill(g) }\end{array}$ & $\begin{array}{c}\text { Tuber yield } \\
\text { /plot(kg) }\end{array}$ & $\begin{array}{l}\text { No. of tuber } \\
\text { /plot }\end{array}$ \\
\hline \multirow{12}{*}{$\mathrm{G}_{2}$} & Diamant & $87.00 \mathrm{a}-\mathrm{c}$ & $267.4 \mathrm{e}-\mathrm{j}$ & 23.28 a-d & 448.7 h-m \\
\hline & Patrones & $65.00 \mathrm{f}-\mathrm{h}$ & $312.0 \mathrm{~d}-\mathrm{f}$ & $21.28 \mathrm{c}-\mathrm{g}$ & $849.7 \mathrm{a}$ \\
\hline & Raja & $25.67 \mathrm{i}$ & $724.0 \mathrm{c}$ & $18.63 \mathrm{~g}-1$ & $508.3 \mathrm{e}-\mathrm{i}$ \\
\hline & BARI TPS1 & $89.00 \mathrm{ab}$ & $239.3 \mathrm{~h}-\mathrm{j}$ & $20.61 \mathrm{~d}-\mathrm{h}$ & $638.0 \mathrm{bc}$ \\
\hline & Arinda & $69.00 \mathrm{e}-\mathrm{g}$ & $339.0 \mathrm{~d}$ & $23.91 \mathrm{a}-\mathrm{c}$ & $529.3 \mathrm{~d}-\mathrm{h}$ \\
\hline & Provento & $82.00 \mathrm{bc}$ & $297.1 \mathrm{~d}-\mathrm{h}$ & $22.83 \mathrm{a}-\mathrm{e}$ & $469.7 \mathrm{~g}-1$ \\
\hline & Felsina & $60.67 \mathrm{~h}$ & $336.1 \mathrm{~d}$ & $19.77 \mathrm{f}-\mathrm{j}$ & $422.3 \mathrm{i}-\mathrm{n}$ \\
\hline & Multa & 72.33 ef & $268.0 \mathrm{e}-\mathrm{j}$ & $18.40 \mathrm{~g}-1$ & $478.3 \mathrm{f}-\mathrm{k}$ \\
\hline & Asterix & $90.00 \mathrm{a}$ & $308.1 \mathrm{~d}-\mathrm{g}$ & $25.13 \mathrm{ab}$ & $627.7 \mathrm{bc}$ \\
\hline & Granola & $90.00 \mathrm{a}$ & $298.1 \mathrm{~d}-\mathrm{h}$ & $25.64 \mathrm{a}$ & $680.7 \mathrm{~b}$ \\
\hline & Cardinal & $70.33 \mathrm{e}-\mathrm{g}$ & $329.8 \mathrm{de}$ & $22.04 \mathrm{c}-\mathrm{f}$ & $510.0 \mathrm{e}-\mathrm{i}$ \\
\hline & Ailsa & $13.00 \mathrm{j}$ & $889.3 \mathrm{a}$ & $11.47 \mathrm{~m}$ & $376.3 \mathrm{lmn}$ \\
\hline \multirow{12}{*}{$\mathrm{G}_{3}$} & Diamant & $86.00 \mathrm{a}-\mathrm{c}$ & $223.9 \mathrm{ij}$ & $18.94 \mathrm{~g}-\mathrm{k}$ & $484.7 \mathrm{f}-\mathrm{k}$ \\
\hline & Patrones & $64.33 \mathrm{gh}$ & $334.7 \mathrm{~d}$ & $20.82 \mathrm{~d}-\mathrm{h}$ & $616.0 \mathrm{~b}-\mathrm{d}$ \\
\hline & Raja & $25.00 \mathrm{i}$ & $702.2 \mathrm{c}$ & $16.27 \mathrm{kl}$ & $378.31-n$ \\
\hline & BARI TPS1 & $87.67 \mathrm{a}-\mathrm{c}$ & $217.4 \mathrm{j}$ & $18.44 \mathrm{~g}-1$ & $468.0 \mathrm{~g}-1$ \\
\hline & Arinda & $68.33 \mathrm{e}-\mathrm{g}$ & $311.0 \mathrm{~d}-\mathrm{g}$ & $19.77 \mathrm{f}-\mathrm{j}$ & $401.7 \mathrm{k}-\mathrm{n}$ \\
\hline & Provento & $81.67 \mathrm{bc}$ & $286.3 \mathrm{~d}-\mathrm{i}$ & 22.48 b-f & $408.7 \mathrm{j}-\mathrm{n}$ \\
\hline & Felsina & $60.00 \mathrm{~h}$ & $263.1 \mathrm{f}-\mathrm{j}$ & 15.971 & 326.0 no \\
\hline & Multa & $74.33 \mathrm{de}$ & $258.0 \mathrm{f}-\mathrm{j}$ & $17.24 \mathrm{i}-1$ & $379.31-n$ \\
\hline & Asterix & $89.33 \mathrm{ab}$ & $260.0 \mathrm{f}-\mathrm{j}$ & $22.24 b-f$ & $582.0 \mathrm{c}-\mathrm{e}$ \\
\hline & Granola & $89.33 \mathrm{ab}$ & $246.1 \mathrm{~g}-\mathrm{j}$ & $22.00 \mathrm{c}-\mathrm{f}$ & $503.7 \mathrm{e}-\mathrm{j}$ \\
\hline & Cardinal & $70.67 \mathrm{e}-\mathrm{g}$ & $311.5 \mathrm{~d}-\mathrm{g}$ & $19.88 \mathrm{e}-\mathrm{i}$ & $431.3 \mathrm{i}-\mathrm{m}$ \\
\hline & Ailsa & $13.00 \mathrm{j}$ & $807.9 \mathrm{~b}$ & $10.53 \mathrm{~m}$ & $246.0 \mathrm{o}$ \\
\hline \multirow{12}{*}{$\mathrm{G}_{4}$} & Diamant & $85.33 \mathrm{a}-\mathrm{c}$ & $241.3 \mathrm{~h}-\mathrm{j}$ & 20.52 d-h & $379.31-n$ \\
\hline & Patrones & $63.67 \mathrm{gh}$ & $329.8 \mathrm{de}$ & $20.72 \mathrm{~d}-\mathrm{h}$ & $570.0 \mathrm{c}-\mathrm{f}$ \\
\hline & Raja & $25.33 \mathrm{i}$ & $713.2 \mathrm{c}$ & $18.08 \mathrm{~h}-1$ & $361.0 \mathrm{mn}$ \\
\hline & BARI TPS1 & $87.67 \mathrm{a}-\mathrm{c}$ & $226.9 \mathrm{ij}$ & $19.70 \mathrm{f}-\mathrm{j}$ & $487.3 \mathrm{e}-\mathrm{k}$ \\
\hline & Arinda & $69.33 \mathrm{e}-\mathrm{g}$ & $337.7 \mathrm{~d}$ & $22.36 \mathrm{~b}-\mathrm{f}$ & $359.7 \mathrm{mn}$ \\
\hline & Provento & $80.67 \mathrm{~cd}$ & $286.0 \mathrm{~d}-\mathrm{i}$ & $22.62 b-f$ & $412.7 \mathrm{i}-\mathrm{n}$ \\
\hline & Felsina & $57.67 \mathrm{~h}$ & $301.2 \mathrm{~d}-\mathrm{h}$ & $17.30 \mathrm{i}-1$ & 392.7 k-n \\
\hline & Multa & $68.33 \mathrm{e}-\mathrm{g}$ & $253.9 \mathrm{f}-\mathrm{j}$ & $16.88 \mathrm{j}-1$ & $374.3 \mathrm{lmn}$ \\
\hline & Asterix & $88.00 \mathrm{a}-\mathrm{c}$ & $262.4 \mathrm{f}-\mathrm{j}$ & $22.70 \mathrm{~b}-\mathrm{f}$ & 415.7 i-n \\
\hline & Granola & $89.00 \mathrm{ab}$ & $286.7 \mathrm{~d}-\mathrm{i}$ & $25.02 \mathrm{ab}$ & $556.0 \mathrm{c}-\mathrm{g}$ \\
\hline & Cardinal & $69.67 \mathrm{e}-\mathrm{g}$ & $309.5 \mathrm{~d}-\mathrm{g}$ & $22.52 b-f$ & $415.3 \mathrm{i}-\mathrm{n}$ \\
\hline & Ailsa & $13.33 \mathrm{j}$ & $854.7 \mathrm{ab}$ & $11.44 \mathrm{~m}$ & $265.0 \mathrm{o}$ \\
\hline \multicolumn{2}{|c|}{$\mathrm{CV}(\%)$} & 5.98 & 8.96 & 10.82 & 10.82 \\
\hline
\end{tabular}

Figures with the same letter(s) in a column are not significantly different at 5\% level. 


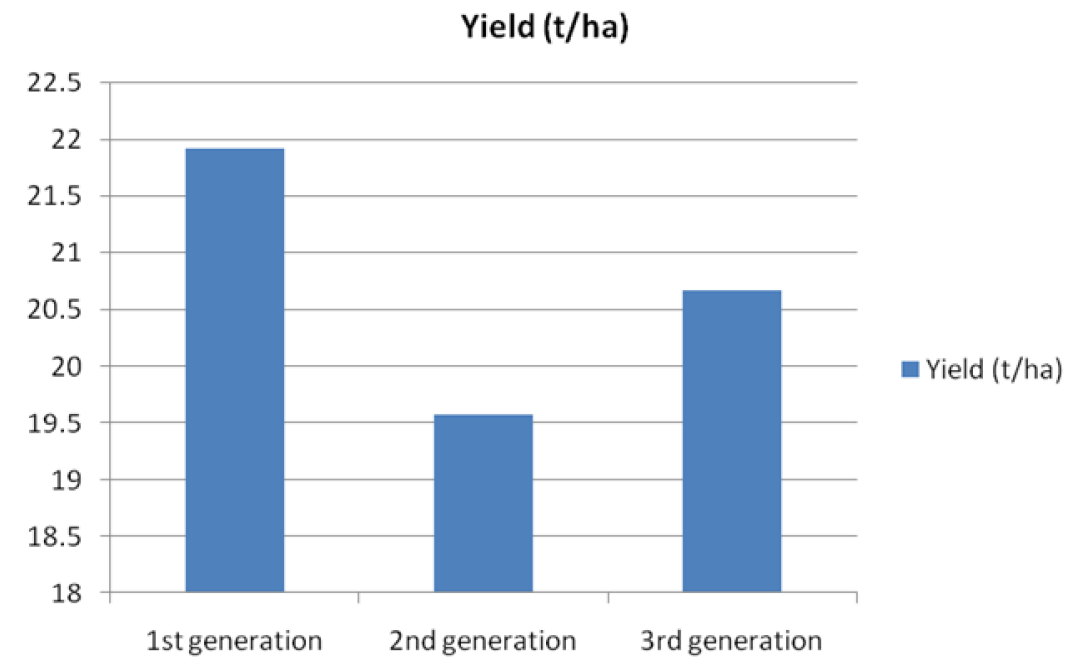

Figure 1. Effect of generations on yield (t/ha) of twelve potato varieties

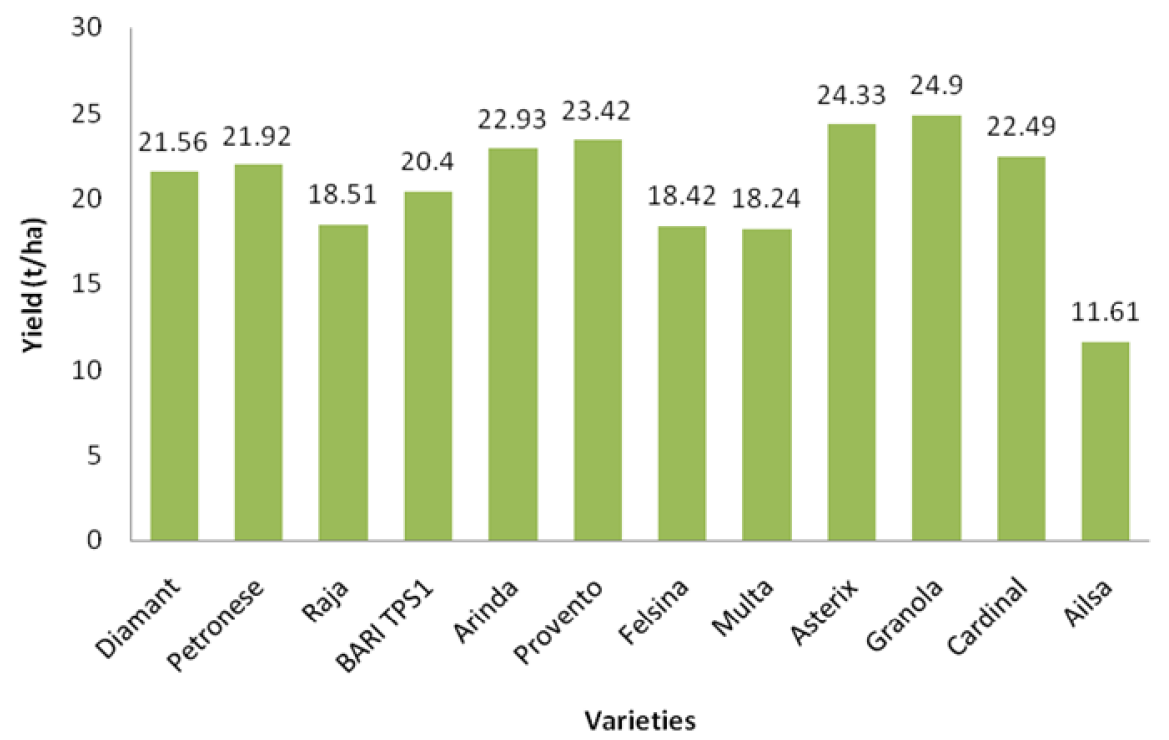

Figure 2. Yields of potato varieties (t/ha) 
Table 9. Interaction effect of variety and generations on yield potential of potato

\begin{tabular}{|c|c|c|c|c|c|c|c|c|}
\hline \multicolumn{5}{|c|}{ Yield (ton/ha) } & \multicolumn{4}{|c|}{$\begin{array}{c}\% \text { increase or decrease over } 1^{\text {st }} \\
\text { generation }\end{array}$} \\
\hline Variety & BS gen. & FS gen. & CS gen. & *Mean & $\begin{array}{l}\text { For FS } \\
\text { gen. }\end{array}$ & $\begin{array}{l}\text { For CS } \\
\text { gen. }\end{array}$ & Mean & $\begin{array}{l}\text { Diseased } \\
\text { Plant }(\%)\end{array}$ \\
\hline Diamant & $23.58 \mathrm{a}-\mathrm{d}$ & $19.73 \mathrm{~g}-\mathrm{j}$ & $21.38 \mathrm{~d}-\mathrm{h}$ & 21.56 & -16.33 & -9.33 & -12.83 & 14.70 \\
\hline Petrones & $22.50 \mathrm{c}-\mathrm{f}$ & $21.69 \mathrm{~d}-\mathrm{g}$ & $21.58 \mathrm{~d}-\mathrm{g}$ & 21.92 & -3.60 & -4.09 & -3.84 & 23.83 \\
\hline Raja & $19.74 \mathrm{~g}-\mathrm{j}$ & $16.95 \mathrm{k}$ & 18.83 h-k & 18.51 & -14.13 & -4.42 & -9.37 & 16.75 \\
\hline BARI TPS1 & 21.47d-h & $19.21 \mathrm{~g}-\mathrm{k}$ & $20.52 \mathrm{f}-\mathrm{i}$ & 20.40 & -10.53 & -4.61 & -7.48 & 30.16 \\
\hline Arinda & $24.91 \mathrm{a}-\mathrm{c}$ & 20.59 e-i & $23.29 \mathrm{c}-\mathrm{e}$ & 22.93 & -17.34 & -6.50 & -11.92 & 29.09 \\
\hline Provento & $23.78 a-d$ & $23.42 \mathrm{~b}-\mathrm{d}$ & $23.06 \mathrm{c}-\mathrm{f}$ & 23.42 & -1.51 & -3.03 & -2.27 & 22.77 \\
\hline Felsina & $20.60 \mathrm{e}-\mathrm{i}$ & $16.64 \mathrm{k}$ & $18.02 \mathrm{i}-\mathrm{k}$ & 18.42 & -19.22 & -12.52 & -15.87 & 26.66 \\
\hline Multa & $19.17 \mathrm{~g}-\mathrm{k}$ & $17.96 \mathrm{i}-\mathrm{k}$ & $17.59 \mathrm{jk}$ & 18.24 & -6.31 & -8.24 & -7.28 & 30.52 \\
\hline Asterix & $26.18 \mathrm{a}$ & $23.17 \mathrm{c}-\mathrm{f}$ & $23.65 \mathrm{a}-\mathrm{d}$ & 24.33 & -11.50 & -9.66 & -10.58 & 23.83 \\
\hline Granola & $26.04 \mathrm{ab}$ & $23.92 \mathrm{a}-\mathrm{d}$ & $24.73 \mathrm{a}-\mathrm{c}$ & 24.90 & -8.14 & -5.03 & -6.59 & 6.95 \\
\hline Cardinal & $23.29 \mathrm{c}-\mathrm{e}$ & $20.71 \mathrm{e}-\mathrm{h}$ & $23.46 \mathrm{~b}-\mathrm{d}$ & 22.49 & -11.08 & +0.73 & -5.17 & 24.25 \\
\hline Ailsa & 11.941 & 10.961 & 11.921 & 11.61 & -8.21 & -0.17 & -4.19 & 27.00 \\
\hline $\mathrm{CV}(\%)$ & \multicolumn{4}{|c|}{6.74} & & & & \\
\hline $\mathrm{LSD}_{0.05}$ & \multicolumn{4}{|c|}{2.275} & & & & \\
\hline Mean & $21.93 \mathrm{~A}$ & $19.58 \mathrm{C}$ & $20.67 \mathrm{~B}$ & & & & & \\
\hline
\end{tabular}

Figure with the same letter(s) in a column are not significantly different at 5\% level.

*Average of 3 generations

The average yield of $3^{\text {rd }}$ generation was significantly lower $(19.58 \mathrm{t} / \mathrm{ha})$ than that of $2^{\text {nd }}$ generation $(21.93 \mathrm{t} / \mathrm{ha})$, this might be due to degeneration caused by viral diseases although the reduction of yield in the $3^{\text {rd }}$ generation over the $2^{\text {nd }}$ generation occurred in all varieties, the degree of reduction varied widely. It was the lowest in Provento (-1.51\%) followed by Patrones $(-3.6 \%)$ and Multa $(-6.31 \%)$ and the highest in Felsina $(-19.22 \%)$ followed Arinda $(-17.34 \%)$, Diamant (-16.33\%) and Raja $(-14.13 \%)$ (Table 9). High reduction of yield in the $3^{\text {rd }}$ generation over $2^{\text {nd }}$ generation indicated that these varieties were more susceptible to degenerative diseases (Table 9). The average yield of $4^{\text {th }}$ generation was lower $(20.67 \mathrm{t} / \mathrm{ha})$ than $2^{\text {nd }}$ generation but higher than $3^{\text {rd }}$ generation. In the $4^{\text {th }}$ generation the yield ranged from 11.92 to 24.73 tons per hectare. All the varieties, except Provento and Multa, showed slightly increase in yield than the $3^{\text {rd }}$ generation. This might be due to favorable environment in the $4^{\text {th }}$ generation although disease infection was higher than other generations.

The rate of yield reduction is calculated as percent yield reduction in successive generations over $2^{\text {nd }}$ generation. Considering all the three generations, the highest reduction in yield was observed in Felsina $(-15.87 \%)$ followed by Diamant (-12.83\%), Arinda (-11.92\%), Asterix $(-10.58 \%)$ and Raja $(-9.37 \%)$ and the lowest was in Provento $(-2.27 \%)$. Granola, Asterix, Provento, Arinda and Cardinal showed better performance in respect of yield in different generations indicating high yield potential. Arinda, Felsina, Asterix and Granola gave good yield in $2^{\text {nd }}$ generation but the rate of yield reduction was very high in the subsequent generations. 
Table 10. Incidence of PVY and PLRV in the three generations as detected by DAS-ELISA

\begin{tabular}{|c|c|c|c|c|c|c|c|c|c|c|c|c|}
\hline \multirow[t]{3}{*}{ Variety } & \multicolumn{12}{|c|}{ Infected plants (Percent) } \\
\hline & \multicolumn{3}{|c|}{$1^{\text {st }}$ generation } & \multicolumn{3}{|c|}{$2^{\text {nd }}$ generation } & \multicolumn{3}{|c|}{$3^{\text {rd }}$ generation } & \multicolumn{3}{|c|}{ Mean of three gen. } \\
\hline & PVY & PLRV & Mixed & PVY & PLRV & Mixed & PVY & PLRV & Mixed & PVY & PLRV & Mixed \\
\hline Diamant & 4.12 & 0.00 & 0.00 & 15.00 & 0.00 & 5.13 & 25.00 & 0.00 & 5.64 & 14.71 & 0.00 & 3.59 \\
\hline Patrones & 5.34 & 1.57 & 0.00 & 21.21 & 2.15 & 0.00 & 34.12 & 7.12 & 0.00 & 20.22 & 3.61 & 0.00 \\
\hline Raja & 6.12 & 0.00 & 0.00 & 15.00 & 0.00 & 0.00 & 25.00 & 4.13 & 2.35 & 15.37 & 1.38 & 0.78 \\
\hline BARI TPS1 & 8.69 & 2.12 & 0.00 & 20.00 & 5.68 & 16.67 & 42.00 & 12.00 & 4.17 & 23.56 & 6.60 & 6.94 \\
\hline Arinda & 8.24 & 2.00 & 0.00 & 25.37 & 5.00 & 9.50 & 40.00 & 6.68 & 8.00 & 24.54 & 4.56 & 5.83 \\
\hline Provento & 7.98 & 2.35 & 0.00 & 15.00 & 8.00 & 0.00 & 25.00 & 10.00 & 0.00 & 15.99 & 6.78 & 0.00 \\
\hline Felsina & 5.98 & 2.08 & 0.00 & 20.98 & 4.17 & 0.00 & 37.78 & 9.00 & 2.13 & 21.58 & 5.08 & 0.71 \\
\hline Multa & 8.12 & 2.10 & 4.17 & 21.87 & 12.50 & 0.00 & 35.98 & 11.00 & 0.00 & 21.99 & 8.53 & 1.39 \\
\hline Asterix & 4.14 & 2.08 & 2.08 & 22.08 & 4.17 & 0.00 & 31.13 & 7.89 & 4.17 & 19.12 & 4.71 & 2.08 \\
\hline Granola & 2.15 & 0.00 & 0.00 & 6.59 & 0.00 & 4.17 & 9.13 & 2.98 & 0.00 & 5.96 & 0.99 & 1.39 \\
\hline Cardinal & 5.34 & 0.00 & 0.00 & 21.68 & 3.24 & 0.00 & 37.38 & 5.12 & 0.00 & 21.47 & 2.79 & 0.00 \\
\hline Ailsa & 9.13 & 3.21 & 5.12 & 15.00 & 10.32 & 4.65 & 32.00 & 11.34 & 5.67 & 18.71 & 8.29 & 5.15 \\
\hline
\end{tabular}


Some varieties such as, Provento and Multa performed the best in the third and forth generations as compared to second generation. Hooker (1981) observed that some varieties gave good yield even with virus diseases but Rashid et al. (1986) found that degeneration due to viruses reduces the yield potential of potato varieties, often very rapidly. However, considering the studied parameters and giving emphasis on yield and degenerative trend and also disease incidence (Table 10), the varieties Provento, Granola and Cardinal showed acceptable performance.

\section{Conclusions}

In respect of plant height, Provento scored the highest at 50 DAP in all the three generations. The shortest plants were observed in the variety Arinda. Plant height decreased in all varieties except Raja and Arinda in the third generation as compared to the first generation and it decreased compared to the second generation only in Patrones and Felsina. The highest foliage coverage was found in Asterix (98.33\%), Multa $(91.67 \%)$ and Provento (93.33\%) at 50 DAP in the first, second and third generations, respectively. The highest average plant vigor was observed in Granola followed by Asterix, Provento and BARI TPS1. The highest number of stems per hill was produced by Patrones in all the generations and the lowest by Arinda. The maximum number of hills per plot was recorded in Asterix (90.00) and Granola (90.00) while the minimum was in Ailsa and Raja in all the generations. Higher tuber yield per hill was obtained from Ailsa which was followed by Raja, Arinda, Felsina and Patrones. The minimum tuber yield per hill was recorded from BARI TPS1 which was more or less similar to Diamant, Multa and Asterix. The highest yield of tuber per plot was obtained from Granola (25.64 $\mathrm{kg}$ ) which was followed by Asterix $(25.13 \mathrm{~kg})$, Arinda $(23.91 \mathrm{~kg})$, Diamant $(23.28 \mathrm{~kg})$ and Provento $(22.83 \mathrm{~kg})$ in the first generation. Nearly similar trend was found in the third generation. In the second generation, Provento yielded the highest $(22.48 \mathrm{~kg})$ which was followed by Asterix (22.24 kg), Granola (22.00 $\mathrm{kg}$ ) and Patrones $(20.82 \mathrm{~kg})$. Ailsa produced the minimum yield in all the generations, which was followed by Multa and Raja in the $1^{\text {st }}$ generation, Felsina and Raja in the $2^{\text {nd }}$ generation and Multa and Felsina $(17.30 \mathrm{~kg})$ in the $3^{\text {rd }}$ generation. Among the three generations, higher yield was obtained in the $1^{\text {st }}$ generation, and then it slightly decreased in the $2^{\text {nd }}$ generation and again increased in the $3^{\text {rd }}$ generation except in Patrones and Multa. The yields of different varieties in the $1^{\text {st }}$ generation ranged from $11.94 \mathrm{t} / \mathrm{ha}$ (Ailsa) to $26.18 \mathrm{t} / \mathrm{ha}$ (Asterix) with an average yield of 19.06 tons/ha. However the highest yield potentiality was observed by Asterix followed by Granola, Arinda, Provento and Diamant in the $1^{\text {st }}$ generation.

\section{References}

Anonymous. 2008. FAO Production Year Book. FAO, Rome, Italy. Database at http://apps.fao.org.

Anonymous. 2010. Annual Report, 2009-10. Tuber Crops Research Centre (TCRC), BARI, Joydebpur, Gazipur.

Anonymous. 2010. Statistical Year Book, 200910. Bangladesh Bureau of Statistics (BBS), Dhaka.

Anonymous. 1988. Annual report, 1987-88. Potato Research Centre. Bangladesh Agricultural Research Institute (BARI), Joydebpur, Gazipur.

Chowdhury, E. H. and. Rasul, M. G. 1995. True potato seed research and development in Bangladesh. Proc. of the workshop on National Programme for True Potato Seed in Bangladesh. BARC, Dhaka; 28-37 pp.

Hossain, M., Hossain, M. I., and Dey, T. K., 2010. Disease free seed potato production through seed plot technique at farm level. Annual report 2009-10. Tuber Crops Research Centre, BARI, Joydebur, Gazipur. 147 p. 
Hooker, W. J. 1981. Compendium of potato diseases. American Phytopath. Soc. USA. $125 \mathrm{p}$.

Pushkarnath. 1976. Potato in Sub-tropics. Orient Longman Ltd., New Delhi. 110002, India. 289 p.
Rashid, M. M., Khan, A. L., and Ali, M. S., 1986. Beej Alu Utpadon (In Bangla). Potato Research Centre, BARI, Joydebpur, Gazipur, Bangladesh. 156 p. 